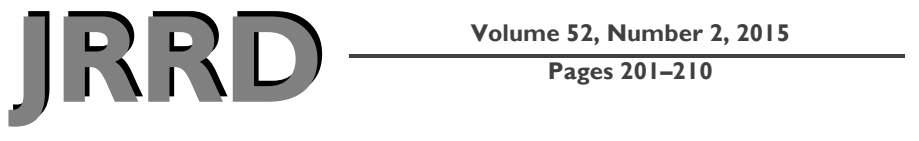

\section{Development and initial validation of the Seated Posture Scale}

\author{
Lelia Barks, PhD, ARNP; ${ }^{*}$ Stephen L. Luther, PhD ${ }^{1}{ }^{\text {Lisa }}$ M. Brown, PhD, ABPP $;{ }^{1-2}$ Brian Schulz, PhD ${ }^{2}$ Mary \\ Elizabeth Bowen, PhD; ${ }^{1,3}$ Gail Powell-Cope, PhD, ARNP ${ }^{1}$ \\ ${ }^{1}$ Center of Innovation for Disability and Rehabilitation Research, James A. Haley Veterans' Hospital, Tampa, FL; and \\ Rehabilitation Research and Development Service, Department of Veterans Affairs (VA), Washington, DC; ${ }^{2}$ School of \\ Aging Studies, University of South Florida, Tampa, FL; ${ }^{3}$ Philadelphia VA Medical Center, Philadelphia, PA; and \\ Center West Chester University, West Chester, PA
}

\begin{abstract}
Literature shows that some health outcomes (e.g., eating, breathing, and speaking) are directly related to posture. Evidence of outcomes mediated by wheelchair seated posture is limited to interface pressure, physical function, and wheelchair skills and safety. This study's purpose was to develop and validate a rapid, low-burden, paper-pencil assessment of wheelchair seated posture for research use and to test feasibility of its use with a sample of older adults. We used a prospective design and a convenience sample of older adults who were receiving rehabilitation services in a community living center. Forty-nine older wheelchair users participated. Main measures were the Seated Posture Scale (SPS), Modified Ashworth Scale, Barthel Index, Visual Descriptor Scale, scale-content validity index (S-CVI), Cronbach alpha, and test-retest reliability. Rating by six experts yielded the overall content validity score (S-CVI) of 0.744 . Total SPS score correlated positively with physical function (Barthel Index, $r=0.46, p<0.001$ ) and negatively with muscle tone (Modified Ashworth Scale, $r=-0.44, p=0.001$ ), supporting SPS construct validity. Internal consistency was 0.66 (Cronbach alpha). Test-retest reliability yielded Pearson product-moment correlations of 0.89 to 0.99 . We conclude that the SPS has sufficient preliminary validity and reliability to support its use as an evaluation of wheelchair seated posture in outcomes research.
\end{abstract}

Key words: elder, geriatric, long-term care, posture, reliability, seat, SPS, validity, Veteran, wheelchair.

\section{INTRODUCTION}

Although seated posture is typically dynamic in nondisabled persons, it is less so for people who require maxi- mum assistance through positioning. Upright positioning in wheelchairs that influences posture affects key health outcomes such as breathing, eating, and pressure ulcer development [1-8]. Posture and outcomes will be poor if the wheelchair is inadequate. Wheelchair seated posture appears to be primarily influenced by three factors: (1) wheelchair configuration; (2) intrinsic postural control, movement, and body characteristics; and (3) positioning by self or a caregiver. More than 70 percent of nursing home residents currently use wheelchairs for seated mobility [9], and many of their wheelchairs were determined to be inadequate [10-12]. Although correct positioning will not fully compensate for inadequate wheelchairs, it will enhance outcomes if appropriate seating is used. These proposed relationships are illustrated as a conceptual framework for wheelchair seated posture in the Figure.

\footnotetext{
Abbreviations: ATD = anthropomorphic test dummy, CLC = community living center, MAS = Modified Ashworth Scale, SCVI = scale-content validity index, SPCM-A = Seated Postural Control Measure for Adults, SPS = Seated Posture Scale, VA = Department of Veterans Affairs, VDS = Visual Descriptor Scale. "Address all correspondence to Lelia Barks, PhD, ARNP; Health Services Research and Development/Rehabilitation Research and Development Center of Innovation for Disability and Rehabilitation Research, 8900 Grand Oak Cir, Tampa, FL 33637; 813-558-3942; fax: 813-558-3990.

Email: Lelia.Barks@va.gov

http://dx.doi.org/10.1682/JRRD.2014.04.0100
} 


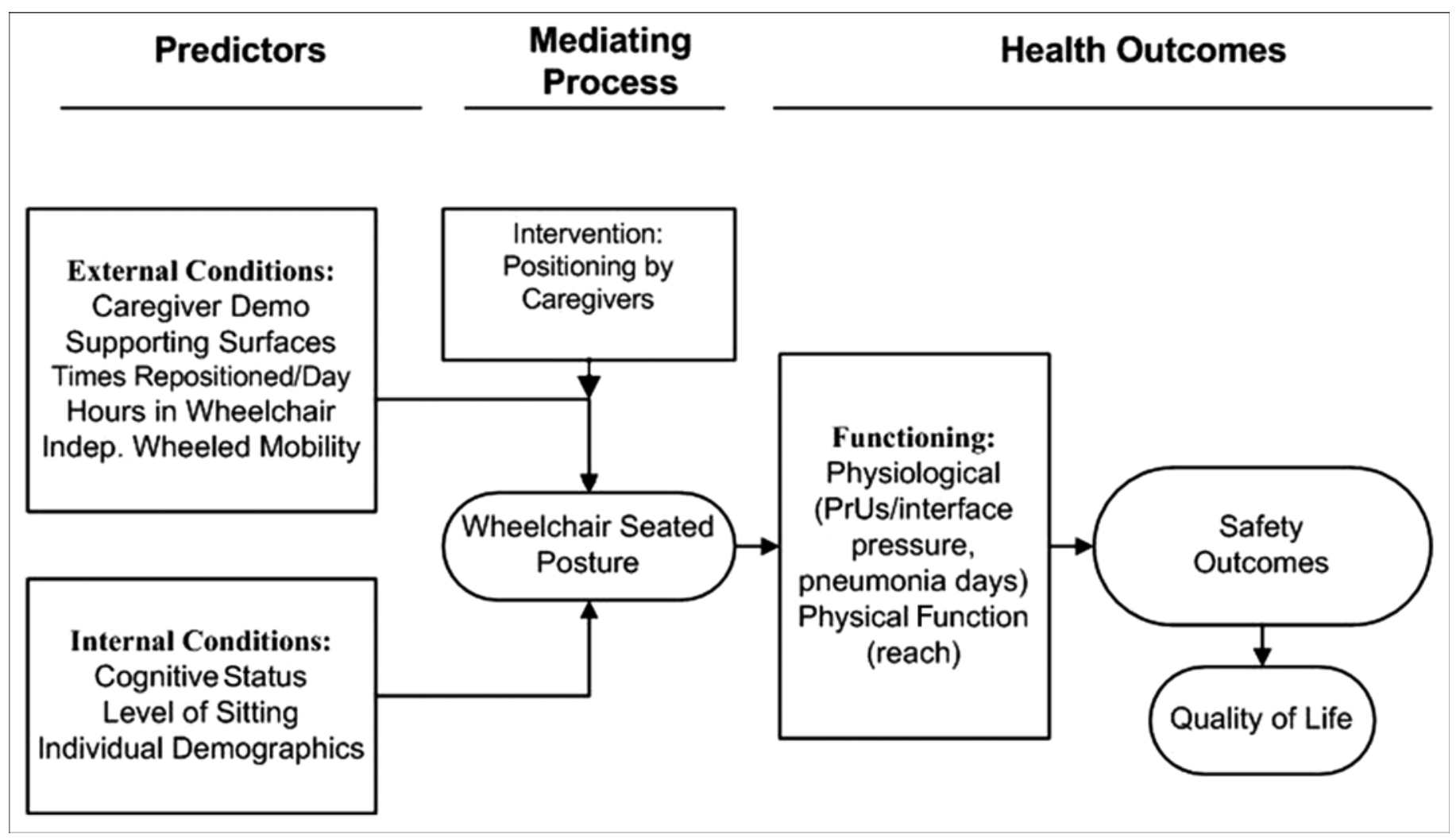

Figure.

Wheelchair seated posture conceptual framework. Indep. = independent, PrUs = pressure ulcers.

Given that rehabilitation settings provide care to people who more likely rely on caregivers for positioning in wheelchairs, it is critical to have empirical evidence to guide positioning [13]. However, currently available methods for evaluating an important outcome of positioning, i.e., wheelchair seated posture, require clinical expertise and use of specialty items, such as a camera, laptop, software, or goniometer [14-15]. Additionally, most existing methods do not evaluate posture of the whole body [14] or are too lengthy to be used efficiently [15] with older adults in long-term care settings. The manual of one validated instrument, the Seated Postural Control Measure for Adults (SPCM-A) [15], which is a two-section, 27-item measure, is currently being translated from French to English. ${ }^{*}$ However, this tool was developed for use by physical

\footnotetext{
*Brigitte Gagnon (Rehabilitation Department, Faculty of Medicine, Laval University, Quebec City, Canada). Conversation with: Lelia Barks (Department of Veterans Affairs Center of Innovation for Disability and Rehabilitation Research, Tampa, Florida). 2013 Oct 17.
}

or occupational therapists and assistive technology practitioners measuring postural control and seated posture in seating systems. The manual has only been available in French; use of a goniometer is required; and although fairly brief, its use in conjunction with other outcome measures is still cumbersome to apply to older adults in the long-term care environment. At present, there are no published, simple, rapidly executable, noninvasive methods requiring little equipment to aid investigators in evaluating overall posture as an outcome mediator of wheelchair seating of older adults. Moreover, in a recent review, Field and Livingstone found no available instrument meeting all criteria for a well-developed measure [16]. Despite proliferation of wheelchair design technology, and except for interface pressure and pressure ulcer development, little is known about the effects of wheelchair seated posture on health outcomes [17]. Intervention research in this area is scant [17]. Most studies focus on wheelchair design, function within the environment (skills), interface pressure, and patient safety. 
There is a need for a valid and reliable, low-burden tool that can be administered rapidly using simple items in the long-term care environment with vulnerable adults. The objective of this study was to develop and provide initial validation data for an instrument that allows evaluation of wheelchair seated posture of older adults in longterm care environments by direct patient care staff. While the initial intent is to use this tool in research applications, in the future it may be modified for clinical use by nursing staff.

\section{METHODS}

\section{Participants}

After obtaining institutional review board approval, we recruited a convenience sample of 49 older adults from a Department of Veterans Affairs (VA) Community Living Center (CLC). Inclusion criteria were English speaking, $>63 \mathrm{yr}$ old, and use of a manual or power wheelchair or scooter at least $6 \mathrm{~h} / \mathrm{d}$. Exclusion criteria were amputation, body weight $>200 \mathrm{lb}$ (dictated by the weight limit of the seating simulator), or spinal cord injury (because of characteristic postures). Prior to study participation, informed consent was obtained.

\section{Seated Posture Scale Instrument Development}

We defined the domain of wheelchair seated posture by adapting the items listed in section 2 (Postural Alignment) of the SPCM-A. Additions were made to the original tool because many older adults who require maximum assistance in seating use their arms to support the upper trunk by leaning on their wheelchair arm supports or, more often, their lap tray. Therefore, we included four items that capture right and left upper-arm sagittal and frontal angles. We also added two lower-limb items: right and left frontal lower-leg angles to obtain additional information regarding leg positioning in the wheelchair. The initial draft included 26 items. After pilot testing, and based on recommendation of our expert panel, two items (pelvic tilt and lumbar curve in the sagittal plane) were deleted because of redundancy and difficulty in reliably scoring the pelvic tilt item. On recommendation of our expert panel, one item (lumbar curve in the frontal plane) was also deleted after the final evaluation round, because of difficulty in reliable scoring and potential redundancy with the pelvic obliquity and trunk lateral shift items. Next, all items were dichotomized, given operational definitions, and organized into a usable scale, with item names conforming to International Organization for Standardization standard 16840-1 [18].

\section{Instruments}

\section{Seated Posture Scale}

The Seated Posture Scale (SPS) is currently a 23-item scale under development, including upper- and lowerlimb positions and trunk positions all related to adult wheelchair seated posture (Appendix, available online only). Each item is dichotomized, using a " 1 " to indicate an anatomical segment aligned in a designated "neutral" wheelchair seated position or a " 0 " to indicate nonalignment of the segment with this designated position. The designated neutral positions are defined as those in which body segments are aligned with either the vertical, horizontal, or wheelchair seat (for transverse angles). A higher summative score for the SPS indicates more body segments are aligned with this designated baseline position. The score does not constitute any type of clinical recommendation for a desired posture.

\section{Prairie Reflections PSS98 Planar Simulator}

We seated the older adult participants in a Prairie Reflections PSS98 Planar Simulator (Prairie Seating Corporation; Skokie, Illinois), on a standard 2 in. foam pressure relief cushion, to standardize seating conditions for participants of varying body sizes.

\section{Modified Ashworth Scale}

The Modified Ashworth Scale (MAS) is a widely used, 6-point ordinal scale used clinically for quantifying muscle tone in persons with neurological injury [19]. Interrater reliability for the MAS was found to be good with a Kendall tau of 0.847 [19].

\section{Barthel Index}

The Barthel Index is a 10-item observational tool measuring ability to perform activities of daily living. Each activity is scored $0-3$ and then summed to generate an overall possible score of 20 [20]. In a recent meta-analysis of overall interobserver reliability, the Barthel Index was found to have a weighted kappa of 0.93 [20].

\section{Visual Descriptor Scale}

The Visual Descriptor Scale (VDS) is an ordinal scale (0-5) that measures intensity of nonmalignant pain, with higher scores indicating more intense pain [21]. 
Concurrent validity of the VDS has been reported as $0.56-0.90$, with test-retest reliability coefficients of 0.75 0.89 [21]. The VDS can be used with older adults with or without cognitive impairments.

\section{Study Procedure}

\section{Content Validity}

We followed Lynn's [22] two-stage process to assess content validity. Development, the first stage, is detailed in the "Seated Posture Scale Instrument Development" section. During the second stage, judgment-quantification, six wheelchair seating experts reviewed content validity of the instrument items and evaluated clarity of each item and instructions for use of the tool. Next, each item was reviewed for relevance to the overall construct of stable, seated posture. Each reviewer awarded " 1 " for clarity and " 0 " for lack of clarity, and " 1 " for relevant and " 0 " for irrelevant. Next, they judged whether the collective items adequately addressed wheelchair seated posture and what items might be missing. Finally, after the study team combined two items and edited all items as needed to enhance clarity according to the experts' initial recommendations, the panel was asked to complete the process again. During the second review round, individual item scores for relevance were averaged and these item means were averaged for the remaining 23 items, according to Davis [23], to obtain the scale-content validity index (S-CVI).

\section{Construct Validity}

All testing was carried out in the VA CLC. After consenting study participants, we configured the seating simulator with a $105^{\circ}$ seat-to-back support angle, a $90^{\circ}$ seat-tolower-leg support angle, and a $90^{\circ}$ lower-leg support-to-foot support angle. The lower-leg support length was adjusted to equal the length measured from the bottom of the thigh at the seat surface to the bottom of the heel of each participant, with the foot in neutral posture $-0^{\circ}$ of dorsiflexion or plantar flexion. We placed arm supports at a height sufficient to allow $90^{\circ}$ of elbow flexion without shoulder protraction or elevation. The seat depth was set at the same depth as the shortest buttock/thigh depth measurement, according to Waugh [24].

With participants seated in the simulator, a single data collector collected all data using the Barthel Index (function), VDS (pain intensity in nonmalignant pain), MAS (muscle tone), and SPS (posture). Muscle tone was scored in the seated position using right and left shoulder, elbow, hip, and knee. A value was selected for each motion (shoulder flexion and extension, ab- and adduction; elbow flexion and extension; hip flexion and extension and hip ab- and adduction in flexion; and knee flexion and extension) at each joint, recording only the highest value for each joint and then summing all eight values to give a total score on muscle tone.

To score the SPS, the tester used a simple metal tape measure and a clear plastic ruler, which provided a $90^{\circ}$ angle to hold up and visualize alignment of body segments along the sides of the $90^{\circ}$ angle.

\section{Test-Retest Reliability}

Initial test-retest reliability of the SPS was evaluated based on data collected in a biomechanics laboratory using three Hybrid III pedestrian anthropomorphic test dummies (ATDs) (Humanetics Innovative Solutions; Plymouth, Michigan). This was done to eliminate the potential for subject movement interfering with a true test of interrater reliability. The ATDs (including 5th, 50th, or 95th percentile anthropometrics) were posed in wheelchairs in seven different seated postures per ATD. Each ATD posture was scored twice using the SPS by four independent raters: one registered nurse, one physical therapist, one assistive technology practitioner, and the principal investigator (a registered nurse practitioner) for a total of 42 trials per rater. An interval of 2 min between trials was selected to maximize efficiency of testing. With the complexity of postures and SPS scale items, the research team felt 2 min was adequate to eliminate any effect of memory.

\section{Internal Item Consistency and Item Analysis}

Data collected in the VA CLC (as described earlier) were used for calculation of internal item consistency and for SPS item analysis tasks. We calculated internal consistency and conducted item analysis of all items of the SPS. Finally, we evaluated feasibility of the SPS by taking the mean of SPS burden (time to score each of 12 CLC participants who were already seated in their own wheelchairs).

\section{Data Analysis}

\section{Content Validity}

We evaluated content validity by averaging the six expert reviewers' relevance scores for each of 24 provisional individual items and then averaging these 24 values in Microsoft Excel (Microsoft Corporation; Redmond, 
Washington) to develop an overall S-CVI (Table 1). We used the clarity item means to revise the items, which were combined or eliminated and renumbered.

\section{Construct Validity}

Other data were analyzed using SAS version 9.2 (SAS Institute; Cary, North Carolina). Descriptive statistics were calculated to determine frequencies of age, sex, race and ethnicity, comorbidities, and scores on Barthel Index, VDS, and MAS. Values on variables associated with posture (physical function [Barthel Index], pain [VDS], and muscle tone [MAS]) were compared to total SPS score using Pearson product-moment correlation coefficients to provide support for preliminary construct validity of the scale.

\section{Internal Consistency Reliability}

Internal consistency reliability of the SPS was examined using SAS version 9.2 to obtain the Cronbach alpha, which represents the extent to which item responses cor- relate with each other and with total scale score. To determine the effect of specific items on the internal reliability values, we conducted item analysis. Item-total correlations were calculated for all items. Through iterative steps, items with negative or very low $(<0.15)$ values were eliminated and the alpha was recalculated.

\section{Test-Retest Reliability}

For data collected in the laboratory using ATDs, Pearson product moment correlations were calculated from scores at two different time points on the summative scores for each of the four raters.

\section{RESULTS}

\section{Sample}

Forty-nine Veterans over 62 yr of age were enrolled, both community and CLC dwelling. All had a primary

Table 1.

Content validity evaluation by expert panel, final round. Score of $1=$ "clear" or "relevant" and score of $0=$ "unclear" or "irrelevant."

\begin{tabular}{|c|c|c|c|c|c|c|c|c|c|c|c|c|c|c|}
\hline \multirow{2}{*}{ Item } & \multicolumn{7}{|c|}{ Clarity Item Score } & \multicolumn{7}{|c|}{ Relevance Item Score (Item-CVI) } \\
\hline & R1 & $\mathbf{R 2}$ & $\mathbf{R 3}$ & R4 & R5 & R6 & Mean & R1 & $\mathbf{R 2}$ & $\mathbf{R 3}$ & $\mathbf{R 4}$ & R5 & R6 & Mean \\
\hline 1 & 1 & 0 & 1 & 1 & 0 & 0 & 0.50 & 1 & 1 & 1 & 1 & 0 & 0 & 0.67 \\
\hline 2 & 1 & 0 & 1 & 1 & 1 & 1 & 0.83 & 1 & 1 & 1 & 1 & 1 & 0 & 0.83 \\
\hline 3 & 1 & 0 & 0 & 1 & 0 & 1 & 0.50 & 1 & 0 & 1 & 0 & 1 & 0 & 0.50 \\
\hline 4 & 1 & 0 & 1 & 1 & 1 & 1 & 0.83 & 1 & 0 & 1 & 1 & 1 & 1 & 0.83 \\
\hline 5 & 1 & 1 & 1 & 1 & 1 & 1 & 1.00 & 1 & 1 & 1 & 1 & 1 & 1 & 1.00 \\
\hline 6 & 1 & 0 & 1 & 0 & 1 & 1 & 0.67 & 1 & 1 & 1 & 1 & 1 & 1 & 1.00 \\
\hline 7 & 1 & 0 & 1 & 1 & 1 & 1 & 0.83 & 1 & 1 & 1 & 1 & 1 & 1 & 1.00 \\
\hline 8 & 1 & 1 & 1 & 1 & 1 & 1 & 1.00 & 0 & 0 & 0 & 1 & 0 & 0 & 0.17 \\
\hline 9 & 1 & 1 & 1 & 1 & 1 & 1 & 1.00 & 0 & 0 & 0 & 1 & 0 & 0 & 0.17 \\
\hline 10 & 1 & 1 & 1 & 1 & 1 & 1 & 1.00 & 0 & 0 & 0 & 1 & 0 & 0 & 0.17 \\
\hline 11 & 1 & 1 & 1 & 1 & 1 & 1 & 1.00 & 0 & 0 & 0 & 1 & 0 & 0 & 0.17 \\
\hline 12 & 1 & 0 & 1 & 1 & 1 & 1 & 0.83 & 1 & 1 & 1 & 1 & 1 & 1 & 1.00 \\
\hline 13 & 1 & 1 & 1 & 1 & 1 & 1 & 1.00 & 1 & 1 & 1 & 1 & 1 & 1 & 1.00 \\
\hline 14 & 1 & 0 & 1 & 1 & 1 & 1 & 0.83 & 1 & 1 & 1 & 1 & 1 & 1 & 1.00 \\
\hline 15 & 1 & 1 & 1 & 1 & 1 & 1 & 1.00 & 1 & 0 & 1 & 1 & 1 & 1 & 0.83 \\
\hline 16 & 1 & 1 & 1 & 1 & 1 & 1 & 1.00 & 1 & 0 & 1 & 1 & 1 & 1 & 0.83 \\
\hline 17 & 1 & 1 & 1 & 1 & 1 & 1 & 1.00 & 1 & 1 & 1 & 1 & 1 & 1 & 1.00 \\
\hline 18 & 1 & 1 & 1 & 1 & 1 & 1 & 1.00 & 1 & 1 & 1 & 1 & 1 & 1 & 1.00 \\
\hline 19 & 1 & 1 & 1 & 1 & 1 & 1 & 1.00 & 1 & 1 & 1 & 1 & 1 & 1 & 1.00 \\
\hline 20 & 1 & 1 & 1 & 1 & 1 & 1 & 1.00 & 1 & 1 & 1 & 1 & 1 & 1 & 1.00 \\
\hline 21 & 1 & 0 & 1 & 1 & 1 & 0 & 0.67 & 1 & 1 & 1 & 0 & 0 & 1 & 0.67 \\
\hline 22 & 1 & 0 & 1 & 1 & 1 & 0 & 0.67 & 1 & 1 & 1 & 0 & 0 & 1 & 0.67 \\
\hline 23 & 1 & 0 & 1 & 1 & 1 & 1 & 0.83 & 1 & 1 & 1 & 0 & 0 & 1 & 0.67 \\
\hline 24 & 1 & 0 & 1 & 1 & 1 & 1 & 0.83 & 1 & 1 & 1 & 0 & 0 & 1 & 0.67 \\
\hline \multicolumn{8}{|c|}{ Mean Scale CVI } & & & & & & & 0.74 \\
\hline
\end{tabular}


diagnosis with five or more comorbidities. Primary diagnoses were diabetic neuropathy $(n=4)$; stroke $(n=6)$; Parkinson disease $(n=2)$; chronic obstructive pulmonary disease $(n=4)$; sciatica $(n=2)$; osteoarthrosis of the spine $(n=2)$; and gastroesophageal reflux disease, rheumatoid arthritis, depression, cervical spondylosis, pulmonary hypertension, peripheral vascular disease, spastic paraparesis, hypothyroidism, muscle weakness, cardiomyopathy, backache, low back pain, avascular necrosis of hips, hip arthralgia, multiple sclerosis, degenerative joint disease, lung neoplasm, and muscular dystrophy (all $n=1$ ). Additional demographic information is in Table 2.

\section{Seated Posture Scale Instrument}

The SPS scale achieved very good content validity (S-CVI) of 0.744. Polit and Beck consider 0.90 excellent [25]. Of the 24 items, 10 received a perfect mean score of 1.0. Mean relevance scores were lowest on four individual items: posture of right and left upper limbs (right and left upper-arm frontal angle and right and left upper-arm sagittal angle), which lowered the S-CVI. The next lowest scores were four lower-limb items: right and left thigh-to-lower-leg angle and right and left lower leg-tofoot angle.

Construct validity of the SPS was supported, with the Barthel Index being positively associated with SPS score $(r=0.46, p<0.001)$ and MAS being inversely associated $(r=-0.44, p=0.001)$. The VDS was not associated with SPS score $(r=0.22, p=0.12)$.

Internal consistency of study participants' values on total SPS score achieved a Cronbach alpha value of 0.66 (raw data are reported in Table 3). When we conducted

Table 2.

Participant demographic data $(n=49)$.

\begin{tabular}{lc}
\hline \multicolumn{1}{c}{ Variable } & Frequency \\
\hline Age (yr) & 10 \\
$60-69$ & 15 \\
$70-79$ & 20 \\
$80-89$ & 4 \\
$90-99$ & \\
Sex & 1 \\
Female & 48 \\
Male & \\
Race & 4 \\
African American & 45 \\
Caucasian & \\
\hline \hline
\end{tabular}

item analysis, four items $(14,15,18$, and 19$)$ had a low or negative association with total SPS score $(r=0.015$, $-0.091,0.020$, and -0.163 , respectively). When these items were removed and analysis was conducted, the Cronbach alpha increased to 0.74 . Test-retest reliability with the original items using ATDs in wheelchairs yielded Pearson product-moment correlations ranging from 0.89 to 0.99 across four raters. The mean time to score the SPS was 6 min.

\section{DISCUSSION}

Through systematic instrument development methods, we developed a valid and reliable tool to assess posture in frail older adults in long-term care settings. We evaluated content and construct validity and estimated internal consistency of items and feasibility of administration. The SPS is different from existing tools because it requires almost no specialty equipment to execute, is brief, and includes upper-limb items in addition to items for the rest of the body.

While the literature review supported the inclusion of upper-limb items, only one expert panelist thought the upper limbs were relevant in evaluating overall posture. We expect persons with decreasing postural control, such as older adults who use wheelchairs, to need to support the upper trunk with their arms; thus, particularly with older adults, the arms may be important contributors to seated posture. Our item analysis supported inclusion of these items in the SPS. A similar outcome was true for the lower-limb items, with mixed support from the panelists for inclusion of the lower limbs: four members considered the lower-limb items relevant and two rated them irrelevant. However, firm foot and lower leg support are often critical components of seated positioning when postural control is decreased. In a long-term care environment, many individuals have lost or are losing postural control and the need for postural support increases. Even though support from our panel members was mixed, we chose to include these items because of their potential clinical relevance in this population.

Regarding construct validity, as expected, the SPS was associated with physical function and muscle tone. Older adults who sat with more of their body segments in neutral alignment would be expected to perform more activities of daily living (physical function), as reflected in the Barthel Index correlation. The MAS was negatively 
Table 3.

Seated Posture Scale raw data with summary scores by participant (ID) and by item.

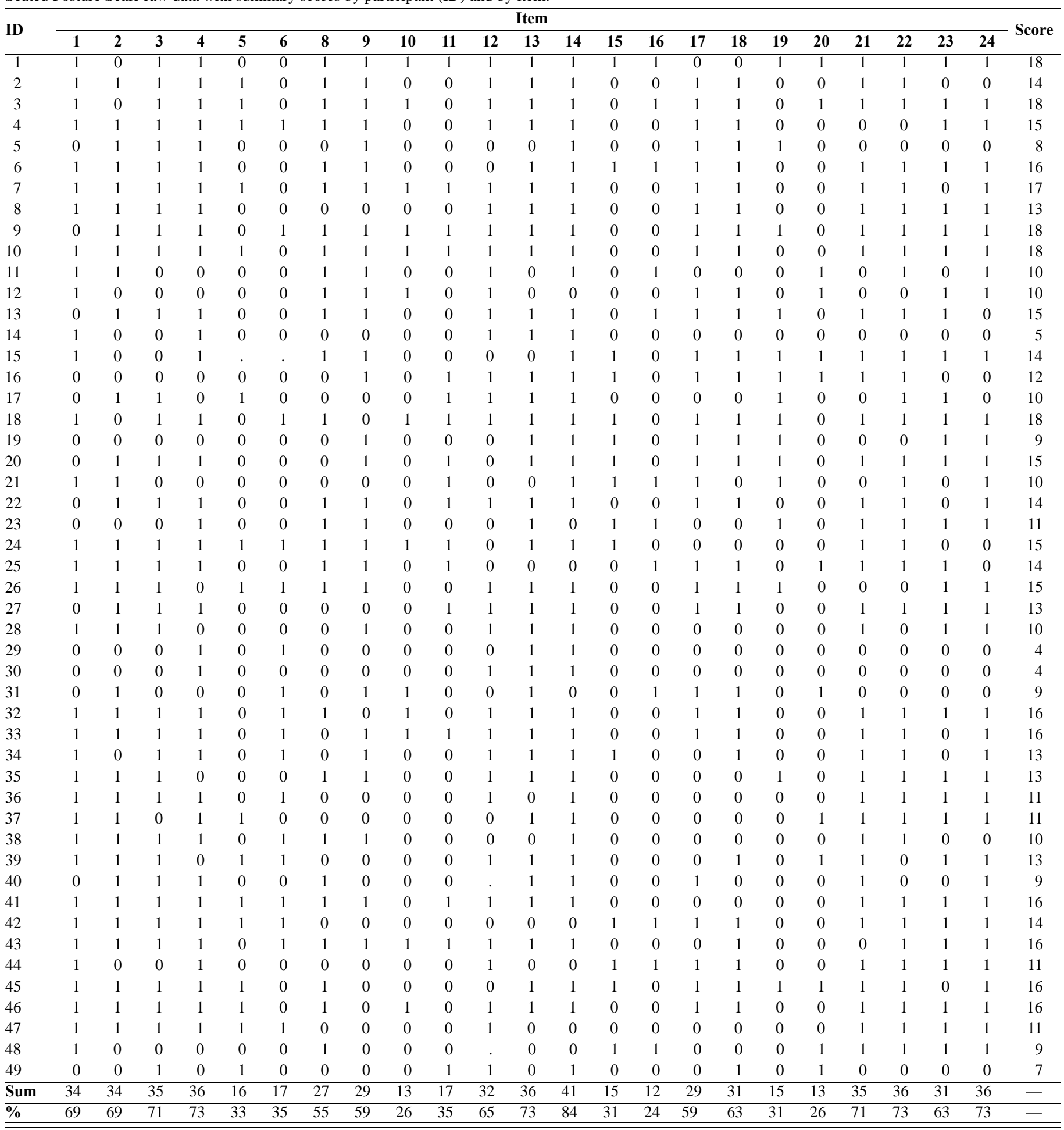

associated with the SPS; that is, the better the posture score, the lower the score on muscle tone and, conversely, the higher the muscle tone, the lower the score on posture.
For example, in conditions like Parkinson disease, where muscles may be rigid, we expect posture to be worse. The VDS was not associated with posture $(r=0.22, p=0.12)$, 
but the VDS is not specific about the type and nature of pain. More information about the nature of pain or discomfort in persons who use wheelchairs might shed light on the association and whether more pain or discomfort is related to poorer posture.

Regarding internal consistency of the SPS, four items with low to negative associations with the total score (numbers 15 and 16, right and left frontal lower-leg angles; and numbers 19 and 20, right and left transverse thigh angles) may represent how older men typically sit. A zero score was assigned if the body segment (lower limb) was not in the designated neutral alignment, which did not allow for any transverse rotation of the thigh on the wheelchair seat. Notably, almost no participants sat with their lower limbs this way, which is reflected in the mean item score for each of these items. It is likely that the designated neutral position of the thighs is not a normal or even an optimal posture for this body segment, at least for the males who predominated in our sample. We are not advocating this designated neutral alignment as optimal posture; it is being used as a designated comparison position for scoring purposes. Additional research will certainly be required to determine postures that are "optimal" for health outcomes in this population. Overall, internal consistency for the SPS was good.

When we considered test-retest reliability for total SPS scores by collecting posture data twice, in addition to collecting data on the outcome variables, our older participants could not maintain the same seated position across all measures without moving. Therefore, we eliminated the issue of movement as a confounder by collecting initial test-retest reliability data using the ATDs in the biomechanics laboratory. Test-retest reliability must still be addressed with human participants.

Of note, we scored alignment in older adults who sit upright in their wheelchairs. None of the 49 study participants was seated with the wheelchair simulator positioned in tilt or recline; however, a number of older adults in long-term care are routinely positioned in their wheelchairs in the tilt or recline position. Items $1,8,9,17,18$, 21 , and 22 cannot be scored using our procedure if the individual is seated in a wheelchair where the seat and/or back are not in an upright position. This is an important issue for older adults who use wheeled mobility, because tilt may actually be an effective solution for improving the posture of older adults. For the purposes of scoring posture, a scale with these items removed may be needed in that particular seating situation and the resulting scale will require further responsiveness testing in a future study.

In scoring items, a small difference (e.g., $5^{\circ}$ ) in ab- or adduction of upper or lower limbs is still scored a " 0 " with the SPS. Therefore, the SPS does not take into account slight variations in posture. Some postures may be out of neutral alignment but comprise "normal" sitting for adults in wheelchairs. Much larger sample sizes are needed to describe normal posture. A positive score of " 1 " on each of the following items constitutes acceptable posture: $90^{\circ}-105^{\circ}$ of hip, elbow, knee, and ankle flexion and $0^{\circ} \mathrm{ab}$ - or adduction at the shoulder and hip. Although completely neutral alignment may not be a normal seated posture, frail older adults seated in wheelchairs may require the support that neutral alignment provides, especially when they cannot reposition themselves. Because this population is already at great risk of poor rehabilitation outcomes in long-term care, poor posture could exacerbate existing conditions, worsen health status, and hinder rehabilitation efforts.

This study was limited in sample size. Given the preliminary nature of the study, future work will need to be conducted to determine the best composition of the SPS with the best set of items. We also recruited only one woman, because of the demographic characteristics of our older Veteran population.

It is possible that in scoring test-retest data with the ATDs, after 2 min out of the room, the scorer could remember the first item decision made, influencing the second score; however, we attempted to achieve rapid scoring of a large number of postures by several raters, and this method was the most feasible we could achieve.

More test-retest reliability testing is needed to address potential error that would be seen in evaluating real people. Future research includes plans to establish criterionrelated validity for the SPS, extend test-retest reliability testing, and include more women participants.

\section{CONCLUSIONS}

This research used an iterative method to develop and evaluate validity and reliability of the SPS, a low-burden, low-technology, rapid evaluation of seated posture in older adults who use wheelchairs for seating and mobility. Support for content validity of the SPS was provided through review by six experts in the field. The total SPS score for the older adults in the study correlated positively with 
physical function (Barthel Index, $r=0.46, p<0.001$ ) and negatively with muscle tone (MAS, $r=-0.44, p=0.001$ ), supporting the construct validity of the SPS. Internal consistency on the SPS score was 0.66, based on Cronbach alpha. Test-retest reliability using ATDs in wheelchairs yielded Pearson product-moment correlations ranging from 0.89 to 0.99 across four raters. These results provide strong preliminary support for the validity and reliability of the SPS. Research is ongoing to further refine the SPS, and future use may include evaluation of wheelchair seated posture in outcomes research and clinical settings.

\section{ACKNOWLEDGMENTS}

\section{Author Contributions:}

Study concept and design: L. Barks, G. Powell-Cope, S. L. Luther. Acquisition of data: L. Barks, B. Schulz, M. E. Bowen.

Analysis and interpretation of data: L. Barks, S. L. Luther, G. Powell-Cope, B. Schulz, L. M. Brown.

Drafting of manuscript: L. Barks, L. M. Brown.

Critical revision of manuscript for important intellectual content:

S. L. Luther, G. Powell-Cope, L. M. Brown.

Statistical analysis: S. L. Luther, L. Barks.

Administrative, technical, or material support: B. Schulz, M. E. Bowen, G. Powell-Cope.

Financial Disclosures: The authors have declared that no competing interests exist.

Funding/Support: This material was based on work supported initially by the VA Office of Academic Affiliations (postdoctoral fellowship) and later by the VA Office of Research and Development, Rehabilitation Research and Development Service (Career Development Award 2 No. 07646-W).

Additional Contributions: We gratefully acknowledge the support of our clinical partners, the Geriatric and Extended Care Service of the James A. Haley Veterans' Hospital, particularly the staff of Haley's Cove CLC, in this study.

Institutional Review: The study protocol was approved by the institutional review board of the University of South Florida. All participants provided written informed consent before participating.

Participant Follow-Up: The authors do not plan to inform participants of the publication of this study.

Disclaimer: This material was presented in a podium presentation at the International Seating Symposium, March 9, 2013, Nashville, Tennessee. Contents do not represent the views of the VA or the U.S. Government.

\section{REFERENCES}

1. Lin F, Parthasarathy S, Taylor SJ, Pucci D, Hendrix RW, Makhsous M. Effect of different sitting postures on lung capacity, expiratory flow, and lumbar lordosis. Arch Phys
Med Rehabil. 2006;87(4):504-9. [PMID:16571389]

http://dx.doi.org/10.1016/j.apmr.2005.11.031

2. McFarland DH, Lund JP, Gagner M. Effects of posture on the coordination of respiration and swallowing. J Neurophysiol. 1994;72(5):2431-37. [PMID:7884469]

3. Nwaobi OM, Smith PD. Effect of adaptive seating on pulmonary function of children with cerebral palsy. Dev Med Child Neurol. 1986;28(3):351-54. [PMID:3721078] http://dx.doi.org/10.1111/j.1469-8749.1986.tb03883.x

4. Logemann JA, Kahrilas PJ, Kobara M, Vakil NB. The benefit of head rotation on pharyngoesophageal dysphagia. Arch Phys Med Rehabil. 1989;70(10):767-71. [PMID:2802957]

5. Jan YK, Jones MA, Rabadi MH, Foreman RD, Thiessen A. Effect of wheelchair tilt-in-space and recline angles on skin perfusion over the ischial tuberosity in people with spinal cord injury. Arch Phys Med Rehabil. 2010;91(11):1758-64. [PMID:21044723] http://dx.doi.org/10.1016/j.apmr.2010.07.227

6. Makhsous M, Priebe M, Bankard J, Rowles D, Zeigler M, Chen D, Lin F. Measuring tissue perfusion during pressure relief maneuvers: Insights into preventing pressure ulcers. J Spinal Cord Med. 2007;30(5):497-507. [PMID:18092567]

7. Sprigle S, Maurer C, Holowka M. Development of valid and reliable measures of postural stability. J Spinal Cord Med. 2007;30(1):40-49. [PMID:17385269]

8. Hobson DA. Comparative effects of posture on pressure and shear at the body-seat interface. J Rehabil Res Dev. 1992;29(4):21-31. [PMID:1432724] http://dx.doi.org/10.1682/JRRD.1992.10.0021

9. Brechtelsbauer DA, Louie A. Wheelchair use among longterm care residents. Ann Longterm Care. 1999;7(6):213-20.

10. Hubbard S, Fitzgerald SG, Reker D, Kazis L, Downs F. Relationship between health related quality of life and quality of wheelchair provided to veterans. Proceedings of the 27th Annual RESNA Conference; 2004 Jun 20-22; Orlando, FL.

11. Wick JY, Zanni GR. Wheelchair-bound residents in nursing facilities: The basics. Consult Pharm. 2007;22(2):119-22, 132-34, 137-39. [PMID:17367246] http://dx.doi.org/10.4140/TCP.n.2007.119

12. Fuchs RH, Gromak PA. Wheelchair use by residents of nursing homes: Effectiveness in meeting positioning and mobility needs. Assist Technol. 2003;15(2):151-63. [PMID:15137732] http://dx.doi.org/10.1080/10400435.2003.10131899

13. Gavin-Dreschnack D, Barks L. Wheelchair positioning and patient positioning clinical practice guidelines. In: Evidencebased nursing care guidelines: Medical-surgical interventions. St. Louis (MO): Mosby-Elsevier; 2008. p. 622-29.

14. Furui T, Furui M, Sakai K, Komatshu T, Fujihira Y, Hisari A, Fujino F, Kagatani M, Handa T. Utility of wheelchair seated posture measurement based on ISO16840-1. Proceedings of 
the 26th Annual International Technology and Persons with Disabilities Conference; 2011; San Diego, CA.

15. Gagnon B, Noreau L, Vincent C. Reliability of the seated postural control measure for adult wheelchair users. Disabil Rehabil. 2005;27(24):1479-91. [PMID:16421073] http://dx.doi.org/10.1080/09638280500276570

16. Field D, Livingstone R. Clinical tools that measure sitting posture, seated postural control or functional abilities in children with motor impairments: A systematic review. Clin Rehabil. 2013;27(11):994-1004. [PMID:23858526] http://dx.doi.org/10.1177/0269215513488122

17. Trefler E, Fitzgerald SG, Hobson DA, Bursick T, Joseph R. Outcomes of wheelchair systems intervention with residents of long-term care facilities. Assist Technol. 2004; 16(1):18-27. [PMID:15357146] http://dx.doi.org/10.1080/10400435.2004.10132071

18. Waugh K, Crane B. A clinical application guide to standardized wheelchair seating measures of the body and seating support surfaces, Revised edition. Wheelchair seating [Internet]. Aurora (CO): University of Colorado School of Medicine Assistive Technology Partners; 2013 Aug 1. Available from: http://www.ucdenver.edu/academics/ colleges/medicalschool/programs/atp/Resources/ WheelchairGuide/Pages/WheelchairGuideForm.aspx

19. Bohannon RW, Smith MB. Interrater reliability of a modified Ashworth scale of muscle spasticity. Phys Ther. 1987; 67(2):206-7. [PMID:3809245]

20. Duffy L, Gajree S, Langhorne P, Stott DJ, Quinn TJ. Reliability (inter-rater agreement) of the Barthel Index for assessment of stroke survivors: Systematic review and meta-analysis. Stroke. 2013;44(2):462-68. [PMID:23299497] http://dx.doi.org/10.1161/STROKEAHA.112.678615

21. Flaherty E. Pain assessment for older adults [Internet]. New York (NY): The Hartford Institute for Geriatric Nurs- ing, New York University, College of Nursing; 2012 [cited 2013 Jul 30]. Available from:

http://consultgerirn.org/uploads/File/trythis/try_this_7.pdf

22. Lynn MR. Determination and quantification of content validity. Nurs Res. 1986;35(6):382-85. [PMID:3640358] http://dx.doi.org/10.1097/00006199-198611000-00017

23. Davis LL. Instrument review: Getting the most from a panel of experts. Appl Nurs Res. 1992;5(4):194-97. http://dx.doi.org/10.1016/S0897-1897(05)80008-4

24. Waugh K. Wheelchair seating for postural control and function. Training program at Children's Hospital, Denver, CO. 2004 Mar.

25. Polit D, Beck C. Assessing measurement quality in quantitative studies. In: Polit D, Beck C. Nursing research: Generating and assessing evidence for nursing practice. 8th ed. Philadelphia (PA): Lippincott; 2008. p. 458-59.

Submitted for publication April 9, 2014. Accepted in revised form January 15, 2015.

This article and any supplementary material should be cited as follows:

Barks L, Luther SL, Brown LM, Schulz B, Bowen ME, Powell-Cope G. Development and initial validation of the Seated Posture Scale. J Rehabil Res Dev. 2015;52(2): 201-10.

http://dx.doi.org/10.1682/JRRD.2014.04.0100

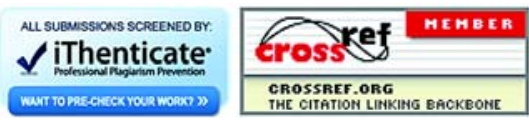

